\title{
Electricity Peak Load Demand using De-noising Wavelet Transform integrated with Neural Network Methods
}

\author{
Pituk Bunnoon
}

Department of Electrical Engineering, Rajamangala University of Technology Srivijaya, Muang Songkhla, Thailand

\begin{tabular}{l} 
Article Info \\
\hline Article history: \\
Received Aug 25, 2015 \\
Revised Nov 3, 2015 \\
Accepted Nov 20, 2015 \\
\hline
\end{tabular}

\section{Keyword:}

De-noisy Wavelet

Mid-term energy demand

Neural network

Peak load forecasts

Weather and economic factors

\begin{abstract}
One of most important elements in electric power system planning is load forecasts. So, in this paper proposes the load demand forecasts using denoising wavelet transform (DNWT) integrated with neural network (NN) methods. This research, the case study uses peak load demand of Thailand (Electricity Generating Authority of Thailand: EGAT). The data of demand will be analyzed with many influencing variables for selecting and classifying factors. In the research, the de-noising wavelet transform uses for decomposing the peak load signal into 2 components these are detail and trend components. The forecasting method using the neural network algorithm is used. The work results are shown a good performance of the model proposed. The result may be taken to the one of decision in the power systems operation.
\end{abstract}

Copyright $(2016$ Institute of Advanced Engineering and Science. All rights reserved.

\section{Corresponding Author:}

Pituk Bunnoon,

Departement of Electrical Engineering,

Faculty of Engineering, Rajamangala University of Technology Srivijaya, Songkhla

1 Ratchadamnoennok Road, Bo Yang, Muang Songkhla Thailand 90000.

Email: dr.pituk.b@ieee.org, Pituk.b@rmutsv.ac.th

$\begin{array}{ll}\text { NOMENCLATURE } \\ \boldsymbol{A I} & \text { Artificial intelligence } \\ \boldsymbol{D W T} & \text { Discret wavelet transforms } \\ \boldsymbol{D N} & \text { De-noisy } \\ \boldsymbol{V} \boldsymbol{S T L F} & \text { Very short term load Forecasting } \\ \boldsymbol{S T L F} & \text { Short term load Forecasting } \\ \boldsymbol{M T L F} & \text { Medium term load Forecasting } \\ \boldsymbol{L T L F} & \text { Long term load Forecasting } \\ \boldsymbol{E G A T} & \text { Electricity Generating Authority of Thailand } \\ \boldsymbol{N N} & \text { Neural network } \\ \boldsymbol{G D P} & \text { Gross domestic product } \\ \boldsymbol{C P I} & \text { Consumer price index } \\ \boldsymbol{T}_{\boldsymbol{m a x}} & \text { Monthly maximum temperature } \\ \boldsymbol{T}_{\boldsymbol{m i n}} & \text { Monthly minimum temperature } \\ \boldsymbol{T}_{\boldsymbol{a v g}} & \text { Monthly average temperature } \\ \boldsymbol{H} & \text { Monthly humidity }\end{array}$

\section{INTRODUCTION}

Peak load demand forecasting, is one of predicted demands for consumption load of the country. It shows a maximum required in each hour, month, and year in the future. Load demand, it is depended on many influencing factors. These factors are economic variables such as industrial factor, consumer price 
index, or GDP of the country, and weather variables for instance temperature, humidity, ultraviolet, rainfall, and wind speed. Thus, the peak load demand forecasting is significantly for the power system operation.

The case of the forecasting can be classified into 4 types such as very short-term, short-term, medium-term, and long-term load forecasts. Each forecasting has advantage as follows: VSTLF result is used for power system operation; STLF result utilizes for power system maintenance and operation; MTLF result uses for fuel reserve planning; LTLF result is related to power plant planning in the future. This research proposes the forecasting interval MTLF model.

In the past of paper, many methods are used for load demand forecasting in the power system. There are two major approaches. These methods are statistic method such as exponential smoothing (ES) [1], multiple linear regression (MLR) [2], and autoregressive integrated moving average (ARIMA) [3-6]. Some methods are conventional approaches. The presented method, artificial intelligence is used a lot in load prediction such as artificial neural network (NN) [7-16], support vector machine (SVM) [17-21], fuzzy logic [22-26]. Many papers at the present propose the forecasting research using wavelet transform with support vector machine or neural network approach [27]. Several papers interest the forecasts in short-term load prediction [28-40], mid-term load forecasts [20] [33], and long-term load forecasting [41-43]. Also, some paper interests to forecast by focus in multi-area forecasts [44] and on the influence of weather factors [32]. While, some researches use AI for other work in power sytem [45-46].

This research presents the load demand forecasts using denoising wavelet transform (DNWT) integrated with neural network (NN) methods. The case study used peak load demand of Thailand (EGAT) is proposed. The peak load demand data will be analysed with influencing variables for selecting and classifying factors. The de-noising wavelet transform will be used for decomposing the monthly peak load signal into two components (detail and trend components) after separating. The main forecast method using the neural network algorithm is used.

This paper is structured as follows. Section 2 provides an introduction to electricity demand and influencing factors of Thailand. Section 3 proposes the theory and implementation methodologies. Section 4 gives the data testing and forecasting results. Section 5 provides the discussion, and finally the conclusion is drawn in section 6.

\section{ELECTRICITY DEMAND AND INFLUENCING FACTORS}

Load characteristics vary significantly among different electricity systems; therefore, it is very important to study the energy demand pattern of the system before constructing the demand forecasting model in the research. In this section, the paper discusses about energy load demand and Influencing factors of the demand in Thailand.
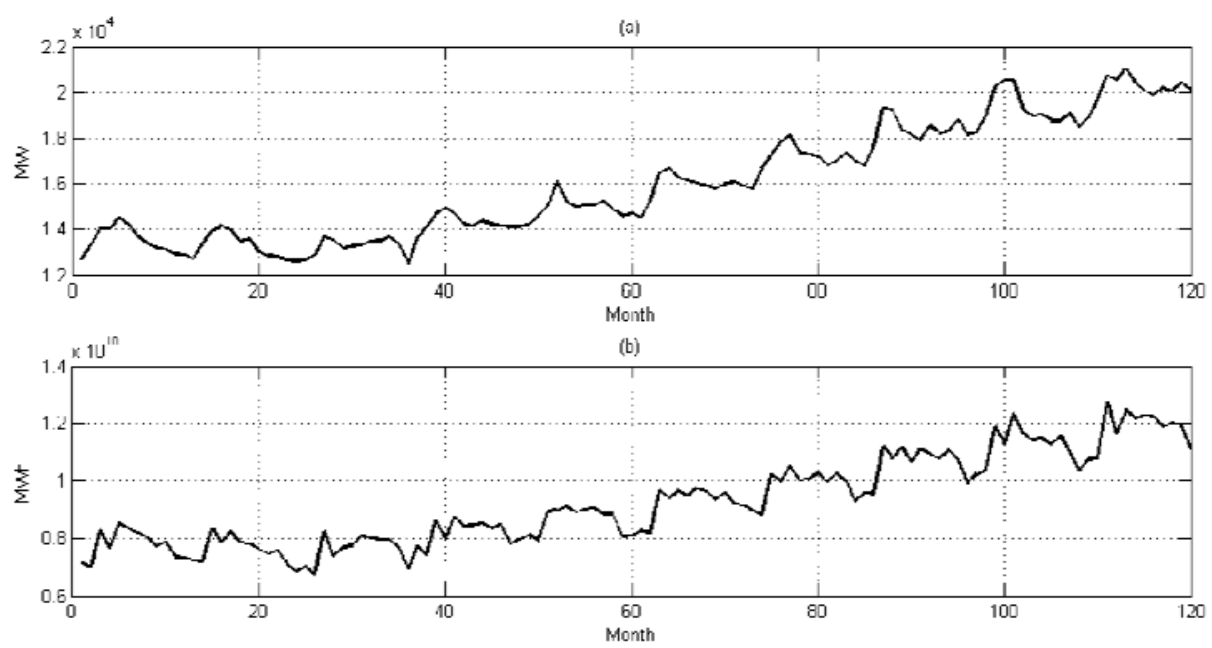

Figure 1. Energy demand (MW) and energy consumption load demand (MWh)

\subsection{Energy Demand}

Electric energy consumption is the form of energy consumption that uses electric energy. It is the actual energy demand made on existing electricity supply. It can be classified into two types that are energy demand $(W)$ and energy consumption demand $(W h)$. The $W$ is a measure of power, while $W h$ is a measure of 
energy. This paper will be explained in MW or megawatt, in Thailand electricity, Electricity Generating Authority of Thailand (EGAT). The peak load demand of the country is shown in Figure 1.

Figure 1, shows two characteristics of energy. There are energy demand $(M W)$ and consumption demand $(M W h)$. These data were recorded from year 1997 to 2004 in monthly type. The electricity energy has the trend of demand to increase every year. Meanwhile, the energy details in all month show the behaviour of peak load demand compliance with influencing factors.

\subsection{Influencing Factors}

In the power system, mid-term load forecasting, the weather and economy are usually the dominant variables in driving the electricity demand of the country. Firstly, the weather factors mean the temperature, humidity, rainfall, and wind speed. The temperature factors for instance maximum temperature, minimum temperature, and average temperature are very affectively in the behaviour component of the demand in each month. It can indicate the customer behaviour caused by the temperature change. For example, especially, if today is higher temperature, the consumers will open air conditioner a lot. It makes more demands of the area or the country. The last, economic variables, there are many factors such as gross domestic product (GDP), consumer price index (CPI), and industrial index (IDI). These economic factors show the economic growth of the country, the growth trend. The significant index is GDP. In summary, both weather and economic influencing factors indicate the load demand of all areas. These factors will affect with the trend of the peak demand and the customer behaviour of each month. So, in the research, both weather and economic factors will be used for the feature input of peak load model forecasting which the feature input is described in the next section.

\section{THEORY AND IMPLEMENTATION METHODOLOGIES}

\subsubsection{De-noisy Wavelet Transforms}

The wavelet transform theory is applicable to several subjects in the research. All wavelet transforms (WT) may be considered pattern form of time-frequency representation for continuous time signals (analog signal) and so are related to harmonic analysis. Almost all practically useful discrete WT use discrete-time filter banks (FB). This FB is called the wavelet and scaling coefficients in wavelet nomenclature. This FB may contain either finite impulse response (FIR) or infinite impulse response (IIR) filters. The wavelets forming a continuous wavelet transform (CWT) are subject to the uncertainly principle of Fourier transform analysis. The product of the uncertainties of time and frequency response scale has a lower bound. Thus, in the scaleogram of a continuous wavelet transform of the signal. Also, discrete wavelet transform (DWT) bases may be considered in the context of other forms of the uncertainly principle. Finally, wavelet transforms are divided into three types: continuous, discrete, and multi-resolution.

Basically, when dealing with wavelet analysis, two types of transforms can be used: the continuous wavelet and the discrete wavelet transforms.

Firstly, continuous wavelet transform (CWT), the continuous wavelet transform is the sum over all time of scaled and shifted value of a wavelet function $\psi$. CWT, when applied on the original signal $f(t)$, is expressed as:

$$
C W T_{-}\{f\}(\tau, s)=\frac{1}{\sqrt{|s|}} \int_{-\infty}^{\infty} f(t) \Psi *\left(\frac{t-\tau}{s}\right) d t
$$

Where $s$ represents the scale parameter, $\tau$ represents the translation parameter, and $\Psi$ represents the wavelet function. Lastly, discrete Wavelet transform (DWT), in practice, signals acquired experimentally are not continuous in time, but sampled as discrete time intervals. Previously, we have seen that the CWT performs a time-frequency resolution (or multiresolution) by scaling (contraction and dilation) and shifting a wavelet function. Recently, it has been shown that such analysis can actually be performed using multiresolution filter banks and wavelet functions, resulting in the Discrete Wavelet Transform (DWT). The DWT is realized by mean of the filters $h[k], g[k]$ that are related to each other through the equation as following.

$$
g[k]=(-1)^{k+1} h[N-k-1]--->k=0,1, \ldots, N-1
$$

Where $N$ represents the length of the signal filter. These filter are constructed from the wavelet kernel $(t)$ and the companion scaling function $\phi(t)$ through the relations below.

Electricity Peak Load Demand using De-noising Wavelet Transform integrated with ... (Pituk Bunnoon) 


$$
\begin{array}{r}
\phi(t)=\sqrt{2} \sum_{k} h[k] \phi(2 t-k) \\
\Psi(t)=\sqrt{2} \sum_{k} g[k] \phi(2 t-k)
\end{array}
$$

Also, de-nosy wavelet transforms the basic idea: one area where wavelets have proven to be very useful is that of nonparametric regression or signal de-noising. This is a way of removing random noise from a series in order to leave the true signal. This is done with no knowledge of the form of the underlying function.

To begin with let us assume that a noisy signal has the form,

$$
y_{i}=g\left(x_{i}\right)+e_{i}
$$

Hence $g\left(x_{i}\right)$ represents the true function to be estimated and $e_{i}$ is some form of random noise usually assumed to be normally distributed. The basic procedure for de-noising is then to take a transform of this noisy signal. The transformed signal will then have the form,

$$
d^{*}=d+\varepsilon
$$

where $d$ represents the transform of the true signal and $\varepsilon$ presents the transform of the noise.

\subsubsection{Neural Network Algorithm}

Neural network algorithm (NN), is an information processing paradigm that is inspired by the way biological nervous systems, such as the brain process information. The key element of this paradigm is the novel structure of the information processing system. It is composed of a large number of highly interconnected processing elements (neurones) working in union to solve specific problems. NN, like people, learn by example. The $\mathrm{NN}$ is configured for a specific application, such as pattern recognition or data classification, through a learning process. Learning in biological system involves adjustments to the synaptic connections that exist between the neurones. This is true of NN as well. Network architecture, in this research, shows multiple-input neuron. Typically, a neuron has more than one input. A neuron with $\mathbf{R}$ inputs is shown in Figure 2. The individual input $p_{1}, p_{2}, \ldots, p_{R}$ are each weighted by corresponding elements $w_{1,1}$, $w_{1,2}, \ldots, w_{1, R}$ of weight matrix $\mathbf{W}$.

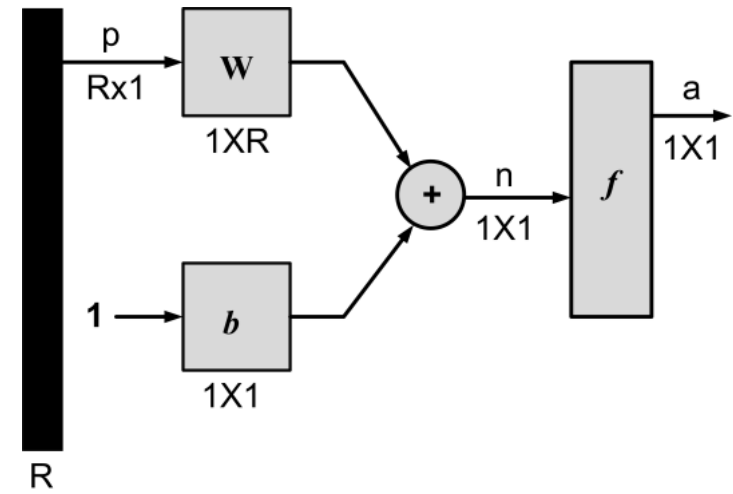

Figure 2. Multiple-Input neural

The neuron has a bias $b$, which is summed with the weighted input to form the net input $n$ :

$$
n=w_{1,1} p_{1}+w_{1,2} p_{2}+\ldots+w_{1 . R} p_{R}+b
$$

This expression can be written in matrix form: 


$$
n=W p+b
$$

where the matrix $\mathrm{W}$ for the signal neurons case has only one row. Now the neuron output can be written as

$$
s=f(W p+b)
$$

This paper uses the neural network for training and forecasting the history peak load demand and prediction in the future. The NN of the model is described in the next section.

\subsubsection{Peak Load Forecasting Model}

In this paper, proposes a new forecasting model. There is the combination of two methods for forecasting. These are de-noising wavelet transforms and artificial neural network approaches. The example model shows in figure 3 which is the de-noisy wavelet transforms integrated with neural network forecasting model based on 3-level. From the figure 3, can be divided into three parts these are the part of the historical data (peak demand and factors), the part of de-noisy wavelet transforms, and neural network part.

Firstly, historical data are recorded from many organizations. There are three major historical factor data. These factors are peak load demand, weather factor, and economic factor.

Secondly, peak load demand preprocessing using de-noisy WT, in this stage use for the peak load demand signal de-noisy before decomposing into 1-level, 2-level, 3-level, 4- level, and 5-level. Each level, both the detail (D) and the trend (A) components are shown.

Finally, this part is forecasting stage using the neural network. It uses three major features. These are peak demand in historical detail and trend components, weather variables, and economic variable. These features are significant data for training and forecasting the peak load demand in the future. After this stage, the forecasting data will be reconstructed to the actual data.

In conclude, the block diagram in figure 3 shows especially the peak load demand decomposition in three-level. It shows major three parts which are significant for model prediction in this research.

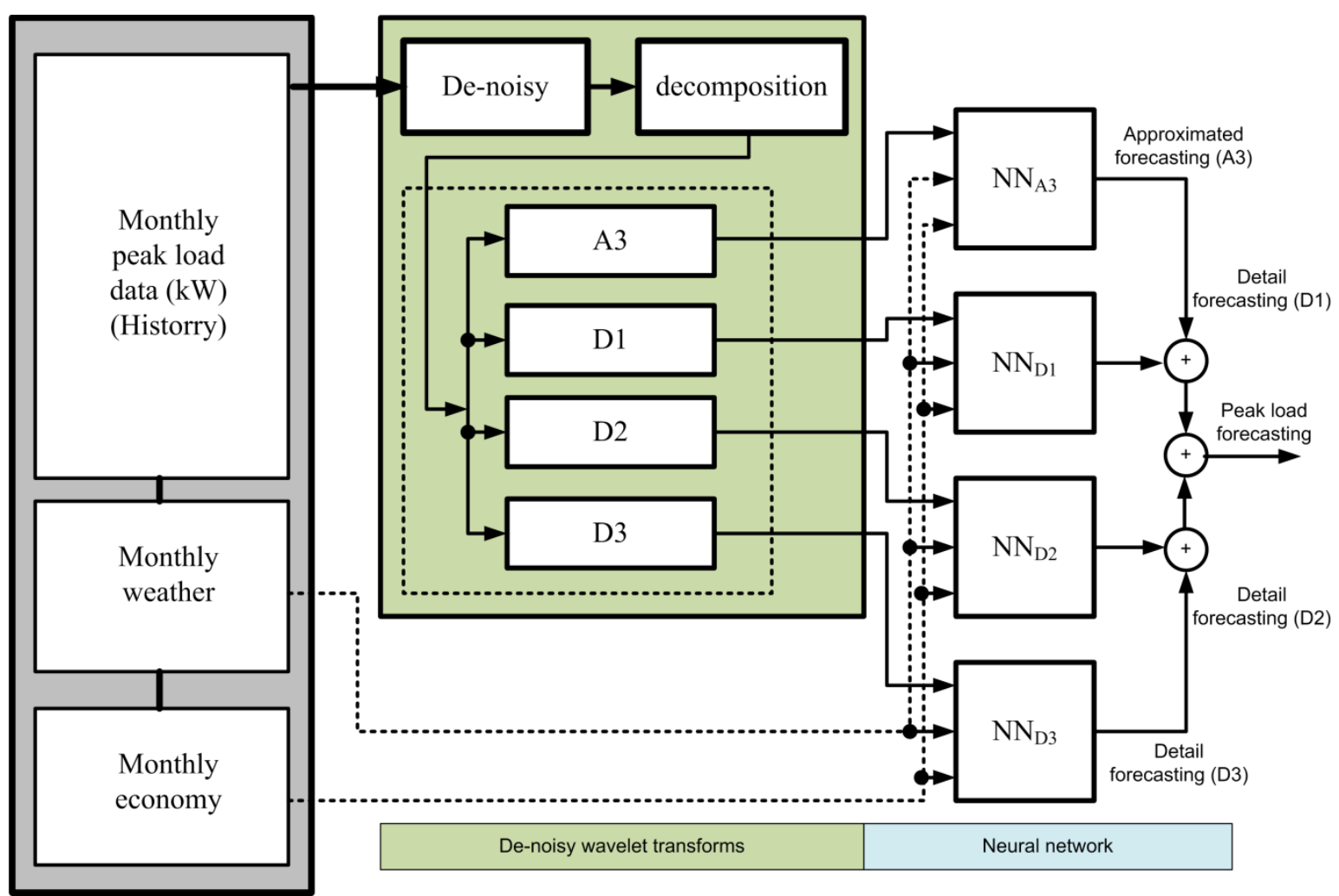

Figure 3. De-noisy wavelet transform integrated with neural network forecasting model based on 3-level 


\section{DATA TESTING AND FORECASTING RESULTS}

The peak load and factors of Thailand country from January 1, 1997 to December 31, 2006 are chosen as the training and testing data for experiments in the research. The data show the recorded in monthly type (January to December). The unit of load demand is in megawatt (MW). Also, in the paper will use the influencing factors such as weather and economic variables of the country in monthly type. The recorded data came from the same year.

In this research, we use the mean absolute percentage error (MAPE) to measure the forecast performance. The MAPE is defined as the ratio between absolute forecasting error and the the actual values of the demand. The MAPE (\%) equations can be calculated as follows.

$$
M A P E=\frac{1}{2} \sum_{1}^{n}\left|\frac{p_{a}-p_{f}}{p a}\right| .100
$$

where $n$ is the number of observation, $p_{a}$ represents the actual peak load demand, and $p_{f}$ represents the forecasting load demand. In Table I, shows the results of peak demand forecasting all 5-level.

The investigative results from figure 3 (for example P3LT) and Table I have the many pattern varies from P1LT to P5LT models. These models come from the number of components in the wavelet transforms decomposition. The neural network approach requires a number of NN model equal to the number of components after WT decomposition. All of the studyresults from 5 models above can be shown in Table I. Table I, in the first column, explains the result of the load forecasting outcome in 12 months from January to December of the year 2007, a period that is forecasted. It uses the five WT models for load prediction. After that, wil compare the results of the level 1, level 2, level 3, level 4, and Level 5 with de-noisy WT. The final results show in the Table I. The P1LT, $\mathrm{P}$ is the maximum load or peak load monthly demand, $1 \mathrm{~L}$ is the 1-level of wavelet transform after signal de-noisy, and $\mathrm{T}$ is the test (testing). Each column will show the peak load forecasting demand in normalized value. It can convert this value to the actual value or peak load value using base value multiplier of the research. Then it can make back to the maximum load value (MW). In the result, the percent error in each month is shown in the PE (\%), which will display all five levels and will finally have the complete the mean absolute percent error (MAPE) of each model. The results are measured by MAPE of each approach, and are compared. In summary, the MAPE of the De-P1LT is 4.39\%. The DeP2LT model, the MAPE is 5.85\%. The MAPE of the De-P3LT is 7.91\%. The De-P4LT, the MAPE is $8.88 \%$. Finally, the De-P5LT, MAPE is 10.72\%, all of which are displayed in the table I. The result will be discussed in the following section.

Table 1. The result of peak load demand forecasting (MW in normalization) in each level

\begin{tabular}{|c|c|c|c|c|c|c|c|c|c|c|}
\hline Level & De-1(P1LT) & & De-2(P2LT) & & De-3(P3LT) & & De-4(P4LT) & & De-5(P5LT) & \\
\hline Month & $\begin{array}{c}\text { Forecasted } \\
(M W)\end{array}$ & PE(\%) & $\begin{array}{c}\text { Forecasted } \\
(M W)\end{array}$ & $\mathrm{PE}(\%)$ & $\begin{array}{c}\text { Forecasted } \\
(M W)\end{array}$ & $\mathrm{PE}(\%)$ & $\begin{array}{c}\text { Forecasted } \\
(M W)\end{array}$ & $\mathrm{PE}(\%)$ & $\begin{array}{c}\text { Forecasted } \\
(M W)\end{array}$ & $\mathrm{PE}(\%)$ \\
\hline January & 0.7266 & -0.05 & 0.7322 & -1.82 & 0.7733 & -7.54 & 0.7600 & -5.69 & 0.7446 & -3.55 \\
\hline February & 0.7249 & 4.64 & 0.7395 & 7.81 & 0.7630 & 4.88 & 0.7642 & 4.73 & 0.7385 & 7.94 \\
\hline March & 0.8254 & 13.82 & 0.7940 & 17.09 & 0.7663 & 19.99 & 0.7584 & 20.82 & 0.7312 & 23.66 \\
\hline April & 0.9999 & 0.01 & 0.8251 & 17.49 & 0.7994 & 20.05 & 0.8028 & 19.71 & 0.7457 & 25.42 \\
\hline May & 0.8336 & 3.71 & 0.8389 & 3.10 & 0.7915 & 8.58 & 0.7793 & 9.98 & 0.7515 & 13.19 \\
\hline June & 0.8247 & 8.12 & 0.8228 & 2.77 & 0.7904 & 11.95 & 0.7772 & 13.42 & 0.7507 & 16.36 \\
\hline July & 0.7972 & -1.86 & 0.8287 & -5.77 & 0.7866 & -.050 & 0.7731 & 1.22 & 0.7546 & 3.58 \\
\hline August & 0.7786 & 5.96 & 0.8092 & 2.27 & 0.7711 & 6.86 & 0.7563 & 8.66 & 0.7473 & 9.74 \\
\hline September & 0.7885 & 7.11 & 0.8011 & 5.62 & 0.7771 & 8.45 & 0.7566 & 10.86 & 0.7521 & 11.39 \\
\hline October & 0.8108 & -1.05 & 0.7837 & 2.31 & 0.7919 & 1.29 & 0.7618 & 5.04 & 0.7621 & 5.00 \\
\hline November & 0.8091 & -2.25 & 0.8054 & -1.78 & 0.8015 & -1.29 & 0.8288 & -4.74 & 0.7769 & 1.81 \\
\hline December & 0.8118 & 3.16 & 0.8184 & 2.37 & 0.8088 & 3.52 & 0.8242 & 1.68 & 0.7794 & 7.02 \\
\hline MAPE & & 4.39 & & 5.85 & & 7.91 & & 8.88 & & 10.72 \\
\hline
\end{tabular}

\section{DISCUSSION}

From the result in the previous section, the forecasts are relatively well during the level 1 and 2 of the de-noisy wavelet transform. The forecasting errors are quite higher in level three, four, and five level of WT decompositions. We conclude that, the de-noisy wavelet for signal separation should be enough made only at one or two level appreciably. Some month, the error is still high because of the weather change is non-stationary in each year, especially, the weather factor of each season. For example, the hot season has very hot for a long time compared with the past. Therefore, further research should study the dynamics of climate data from the past to the present to improve the forecasting results. 


\section{CONCLUSION}

The main contributions made in the present work are presented as follows: in this paper proposes, the load demand forecasting by using de-noising wavelet transform (DNWT) combined with neural network (NN) approach. For this research, the case study of Thailand (Electricity Generating Authority of Thailand: EGAT) is proposed. The peak load data will be analysed with many influencing factors for selecting and classifying factors used in the research. The de-noising wavelet transform is used for decomposing the peak load signal into 2 major components. These components are detail and trend ingredients. Theforecasting method using the neural network method is used. The research results are shown a good performance of the model proposed. The result may be taken to the one of decision in the power systems.

\section{ACKNOWLEDGMENT}

The authors would like to thank the editor and the anonymous reviewers for their valuable comments and suggestions to improve the paper and the presentation.

\section{REFERENCES}

[1] Xianghe Zhu, and Min Shen, "Based on the ARIMA model with grey theory for short term load forecasting model", 2012 International conference on systems and informatics, pp. 564-567, 2012.

[2] Amra N., Ozveren C.C., King D., "Short term load forecasting using multiple linear regression", 42nd International conference of University power engineering, pp. 1192-1198, 2007.

[3] Suhartono, Indah Puspitasari, M. Sjahid Akbar, and Muhammad Hisyam Lee, "Two-level seasonal model based on hybrid ARIMA-ANFIS for forecasting short-term electricity load in Indonesia", 2012 International conference on statistics in science, business and engineering, pp. 1-5, 2012.

[4] Suhartono, Indah Puspitasari, M. Sjahid Akbar, and Muhammad Hisyam Lee, "Two-level seasonal model based on hybrid ARIMA-ANFIS for forecasting short-term electricity load in Indonesia", 2012 International conference on statistics in science, business and engineering, pp. 1-5, 2012.

[5] Xianghe Zhu, and Min Shen, "Based on the ARIMA model with grey theory for short term load forecasting model", 2012 International conference on systems and informatics, pp. 564-567, 2012.

[6] Jian-Chang Lu, Dong-Xiao, and Zheng-Yuan Jia, "A study of shortterm load forecasting based on ARIMA ANN", International conference on machine learning and cybernetics, vol. 5, pp. 3183-3187, 2004.

[7] A.S. Khwaja, M. Naeem, A. Anpalagan, A. Venetsanopoulos, and B. Venkatesh, "Improved short-term load forecasting using bagged neural networks", Electric Power System Research, vol. 125 (2015), pp. 109-115, 2015.

[8] Kenji Nose-Filho, Anna Diva Plasencia, and Carlos Roberto Minussi, "Short-term multinodal load forecasting using a modified general regression neural network", IEEE Trans. Power Del., vol. 26, no. 4, pp. 2862-2869, October 2011.

[9] Osman Z.H., Awad M.L., and Mahmoud T.K., "Neural network based approach for short-term load forecasting", PSCE09 IEEE/PES Power system conference and exposition, pp. 1-8, 2009.

[10] Sajjad Kouhi, Farshid Keynia, and Sajad Najafi Ravadanegh, "A new short-term load forecast method based on neuro-evolutionary algorithm and chaotic feature selection", International Journal of Electrical Power and Energy Systems, vol. 62 (2014), pp. 862-867, November 2014.

[11] Toly Chen, "A collaborative fuzzy-neural approach for long-term load forecasting in Taiwan", Computers and industrial engineering, vol. 62 (2012), pp. 663-670, November 2012.

[12] Sajjad, and Farshid Keynia, "A new cascade NN based method to shortterm load forecast in deregulated electricity market", Energy conversion and management, vol. 71 (2013), pp. 76-83, July 2013.

[13] Yongli Wang, Dongxiao Niu, and Li Ji, "A SOM-based hybrid linearneural model for short-term load forecasting", Neurocomputing, vol. 74, no. 17, pp. 2874-2885, Octorber 2011.

[14] Long-term load forecasting, "Long-term load forecasting based on adaptive neural fuzzy inference system using real energy data", Energy Procedia, vol. 14, pp. 794-799, 2012.

[15] Ruey-Hsun Liang, and Ching-Chi Cheng, "Short-term load forecasting by a neuro-fuzzy based approach", International Journal of Electrical Power and Energy Systems, vol. 24, no. 2, pp. 103-111, February 2002.

[16] Xianghe Zhu, and Min Shen, "Based on the ARIMA model with grey theory for short term load forecasting model", International conference on system and informatics, pp. 564-567, 2012.

[17] Zhongyi Hu, Yukun Bao, and Tao Xiong, "Comprehensive learning particle swarm optimization based memetic algorithm for model selection in short-term load forecasting using support vector regression", Applied Soft Computing, vol. 25 (2014), pp. 15-25, 2014.

[18] Ervin Ceperic, Vladimir Ceperic, and Adrijan Baric, "A strategy for short-term load forecasting by support vector regression machines", IEEE Trans. Power Syst., vol. 28, no. 4, pp. 4356-4364, November 2013.

[19] Rong Gao, Liyuan, and Xiaohua Liu, "Short-term load forecasting based on least square support vector machine combined with fuzzy control", 10th World congress on intelligent control and automation, pp. 1048-1051, 2012.

[20] Xinxing Pan, and Lee B., "A comparison of support vector machines and artificial neural networks for mid-term load forecasting", 2012 IEEE International conference on industrial technology, pp. 95-101, 2012. 
[21] Zhongyi Hu, Yukun Bao, Raymond Chiong, and Tao Xiong, "Midterm interval load forecasting using multi-output support vector regression with a memetric algorithm for feature selection", Energy, vol. 84 (2015), pp. 419-431, May 2015.

[22] Woo-Joo Lee, Jinkyu Hong, "A Hybrid dynamic and fuzzy time series model for mid-term power load forecasting", International Journal of Electrical Power and Energy Systems, vol. 64 (2015), pp. 1057-1062, 2015.

[23] Riswan Efendi, Zuhaimy Ismail, and Mustafa Mat Deris, "A new linguistic out-sample approach of fuzzy time series for daily forecasting of Malaysian electricity load demand", Applied Soft Computing, vol. 28 (2015), pp. 422-430, 2015.

[24] Torkzadeh Roozbeh, Mirzaei Ahmad, Mirjalili Mohammad Mehdi, Anaraki Alireza, Sehhati Mohammad Reza, and Behdad Farideh, "Medium term load forecasting in distribution systems based on multi linear regression and principal component analysis: A novel approach", 2014 19th Conference on electrical power distribution networks, pp. 66-70, 2014.

[25] Toly Chen, and Yu-Cheng Wang, "Long-term load forecasting by a collaborative fuzzy-neural approach", International Journal of Electrical Power and Energy Systems, vol. 43, no. 1, pp. 454-464, December 2012.

[26] Rustum Mamlook, Omar Badran, and Emad Abdulhadi, "A fuzzy inference model for short-term load forecasting", Energy policy, vol. 37, no. 4, pp. 1239-1248, April 2009.

[27] Ming-Yue Zhai, "A new method for short-term load forecasting based on fractal interpretation and wavelet analysis", International Journal of Electrical Power and Energy Systems, vol. 69 (2015), pp. 241-245, 2015.

[28] G. Sudheer, A. Suseelatha, "Short term load forecasting using wavelet transform combined with Holt-Winters and weighted nearest neighbor models", International Journal of Electrical Power and Energy Systems, vol. 64 (2015), pp. 340-346, 2015.

[29] Nian Liu, Qingfeng Tang, Jianhua Zhang, Wei Fan, and Jie Liu, "A Hybrid forecasting model with parameter optimization for short-term load forecasting of micro-grids", Applied Energy, vol. 129 (2014), pp. 336-345, 2014.

[30] Efstathios Paparoditis and Theofanis Sapatinas, "Short-term load forecasting: The similar shape functional timeseries predictor", IEEE Trans. Power Syst., vol. 28, no. 4, pp. 3818-3825, November 2013.

[31] Shu Fan, Luonan Chen, and Wei-Jen Lee, "Short-term load forecasting using comprehensive combination based on multimeteorological information", IEEE Trans. Ind. App., vol. 45, no. 4, pp. 1460-1466, July/August 2009.

[32] Damien Fay, and John V. Ringwood, "On the influence of weather forecast errors in short-term load forecasting models", IEEE Trans. Power Syst., vol. 25, no. 3, pp. 1751-1758, August 2010.

[33] Yang Wang, Qing Xia, and Chongqing Kang, "Secondary forecasting based on deviation analysis for short-term load forecasting", IEEE Trans. Power Syst., vol. 26, no. 2, pp. 500-507, May 2011.

[34] Xiaorong Sun, Peter B. Luh, and Kwok W. Cheung, "An efficient approach for short-term substation load forecasting", 2013 IEEE Power and energy society general meeting, pp. 1-5, 2013.

[35] Adel Abdoos, Mohammad Hemmati, Ali Akbar Abdoos, "Short term load forecasting using a hybrid intelligent method", Knowledge-Based Systems, vol. 76 (2015), pp. 139-147, March 2015.

[36] Pandey A.S., Singh D., Sinha S.K., "Intelligent hybrid wavelet models for short-term load forecasting", IEEE Trans. Power Systems, vol. 25, no. 3, pp. 1266-1273, 2010.

[37] Tao Hong, Pu Wang, and Laura White, "Weather station selection for electric load forecasting", International Journal of Forecasting, vol. 31 (2015), pp. 286-295, 2015.

[38] Liye Xiao, Jianzhou wang, Ru Hou, and Jie Wu, "A combined model based on data pre-analysis and weight coefficients optimization for electrical load forecasting", Energy, vol. 82 (2015), pp. 524-549, 2015.

[39] Gu Chaojun, Dazhi Yang, Panida Jirutitijaroen, Wilfred M. Walsh, and Thomas Reindl, "Spatial load forecasting with communication failure using time-forward kriging", IEEE Trans. Power Syst., vol. 29, no. 6, pp. 2875-2882, November 2014.

[40] Franklin L. Quilumba, Wei-Jen Lee, Heng Huang, David Y. Wang, and Robert L. Szabados, "Using smart meter data to improve the accuracy of intraday load forecasting considering custormer behavior similarities", IEEE Trans. Smart grid, vol. 6, no. 2, pp. 911-918, January 2014.

[41] Zhiheng Zhang, and Shijie Ye, "Long term load forecasting and recommendations for China based on support vector regression", 2011 International conference on information management, innovation management and industrial engineering, pp. 597-602, 2011.

[42] Tao Hong, Wilson J., and Jingrui Xie, "Long term probabilistic load forecasting and normalization with hourly information", IEEE Trans. Smart Grid, vol. 5, no. 1, pp. 456-462, 2014.

[43] Tao Hong, Jason Wilson, and Jingrui Xie, "Long term probabilistic load forecasting and normalization with hourly information", IEEE Trans. Smart grid, vol. 5, no. 1, pp. 456-462, January 2014.

[44] Wen-Chen Chu, Yi-Ping Chen, Zheng-Wei Xu, Wei-Jen Lee, "Multiregion short-term load forecasting in consideration of HI and load/weather diversity", IEEE Trans. Ind. App., vol. 47, no. 1, pp. 232-237, January/ February 2011.

[45] Kamal Yavarian, Farid Hashemi, and Amir Mohammadian, "Design of intelligent PID controller for AVR system using an adaptive neuro-fuzzy inference system”, Internation Journal of Electrical and Computer Engineering, vol. 4, no. 5, October 2014.

[46] Mayadevi N., Vinodchandra S.S., S. Ushakumari, "A review on expert system application is power plants", Internation Journal of Electrical and Computer Engineering, vol. 4, no. 1, February 2014. 


\section{BIOGRAPHY OF AUTHORS}

Pituk Bunnoon was born in Songkhla, Thailand, on September 4, 1973. He received the B.S. in
electrical engineering from Kingmongkut's Institute of Technology Ladkrabang, Thailand, in
2000; M.S.degree in electrical engineering, and the Ph.D. degree in electrical engineering from
Price of Songkla University, Songkhla, Thailand, in 2005 and 2013, respectively. He is currently
working as lecturer in the electrical engineering department of Rajamangala University of
Technology Srivijaya, Songkhla, Thailand. His research interests include applied artificial
intelligence to the power system.

